\title{
Evaluation De La Diversité Phénologique Et Morphologique De Neuf Variétés De Piment (Capsicum Spp.) Cultivées Dans La Région Du Haut-Sassandra (Côte d'Ivoire)
}

\author{
Akaza Moroh Joseph, \\ Goré Bi Boh Nestor, \\ Anzara Gnigouan Kadio Guy Roland, \\ Siniyobo Issa \\ Laboratoire d'amélioration de la production agricole, \\ UFR Agroforesterie, \\ Université Jean Lorougnon Guédé, Côte d’Ivoire
}

Doi:10.19044/esj.2022.v18n03p278

Submitted: 25 August 2021

Accepted: 17 December 2021

Published: 31 January 2022
Copyright 2022 Author(s)

Under Creative Commons BY-NC-ND 4.0 OPEN ACCESS

Cite As:

Akaza M. J., Goré B. B. N., Anzara G. K. G. R. \& Siniyobo I. (2022). Evaluation de la diversité phénologique et morphologique de neuf variétés de piment (Capsicum spp.) cultivées dans la région du Haut-Sassandra (Côte d'Ivoire). European Scientific Journal, ESJ, 18 (3), 278.

https://doi.org/10.19044/esj.2022.v18n03p278

\section{Resume}

La connaissance des caractéristiques des ressources génétiques est nécessaire pour une utilisation efficiente. Or les piments cultivés dans la région du Haut-Sassandra, en Côte d'Ivoire sont insuffisamment caractérisés. Pour déterminer leur diversité phénologique et morphologique, neuf variétés cultivées dans cette région ont été caractérisées à partir de huit paramètres quantitatifs dans un essai en blocs aléatoires complets de neuf traitements avec trois répétitions. Les analyses ont mis en évidence des groupes de variétés aux caractéristiques opposées. Ainsi, une variété, avec le délai de germination le plus court (5 jours), la hauteur de plante la plus élevée $(23 \mathrm{~cm})$, la meilleure croissance latérale, la densité du feuillage la plus grande et la surface foliaire la plus faible (11,62 cm2), s'est nettement distinguée des autres. Aussi, avec un long délai de germination (8 à 9 jours), des plantes de plus faibles diamètres et hauteurs (15 à $16 \mathrm{~cm}$ ), des surfaces foliaires, des nombres de ramifications, des longueurs de ramification la plus développée, des envergures de frondaison et densités de feuillage moyens, quatre variétés se sont distinguées 
des quatre autres variétés présentant des valeurs de paramètres intermédiaires. Les neuf variétés de piment étudiées présentent une importante diversité liée à la germination, la hauteur de plante et la surface foliaire. Les caractéristiques de certaines variétés ont été semblables. Il ressort de ces résultats que la diversité en sein des piments est efficacement mise en évidence avec le délai de germination, la hauteur de la plante, la surface foliaire et peut être exploitée en sélection.

Mots clés: Piment (Capsicum spp.), diversité morphophénologique, HautSassandra, Côte d’Ivoire

\title{
Assessment of the Phenological and Morphological Diversity of Nine Pepper Varieties (Capsicum spp.) Cultivated in the Haut-Sassandra Region (Côte d'Ivoire)
}

\author{
Akaza Moroh Joseph, \\ Goré Bi Boh Nestor, \\ Anzara Gnigouan Kadio Guy Roland, \\ Siniyobo Issa \\ Laboratoire d'amélioration de la production agricole, \\ UFR Agroforesterie, \\ Université Jean Lorougnon Guédé, Côte d’Ivoire
}

\begin{abstract}
Knowledge of the characteristics of genetic resources is necessary for efficient use. However, the peppers cultivated in the Haut-Sassandra region, of Côte d'Ivoire, are insufficiently characterized. To determine their phenological and morphological diversities, nine varieties grown in this region were characterized using eight quantitative parameters in a complete randomized blocks trial of nine treatments with 3 replicates. The analyses revealed groups of varieties with opposite characteristics. Thus, one variety, with the shortest germination time (5 days), the highest plant height $(23 \mathrm{~cm})$, the best lateral growth, the largest foliage density and the lowest leaf area $\left(11.62 \mathrm{~cm}^{2}\right)$ stood out clearly from the others. Also, four varieties, with a long germination time (8 to 9 days), plants of smaller diameters and heights (15 to $16 \mathrm{~cm}$ ), average leaf areas, numbers of branches, length of the most developed branch, canopy size and foliage density differed from the other four varieties with intermediate parameter values. The nine varieties of chilli studied show significant diversity linked to germination, plant height and leaf surface.The characteristics of some varieties are similar. As a consequence of these results,
\end{abstract}


the diversity within peppers is effectively revealed with time to germination, plant height, area of leaf and is to be exploited in breeding.

Keywords: Pepper (Capsicum spp.); Morphophenological diversity, HautSassandra Region, Côte d'Ivoire

\section{Introduction}

Le piment est, actuellement, une culture de grandes exploitations destinée à l'alimentation ordinaire traditionnelle, à l'industrie alimentaire et pharmaceutique. Son utilisation vient de son principe piquant lié à la capsaïcine et de sa richesse en éléments minéraux et en vitamines A et $\mathrm{C}$ (Eching El-Ghoraba et al., 2013). Ses racines, ses feuilles et ses fruits sont consommés en légumes ou condiments. En Côte d'Ivoire, selon Kouassi et Koffi-Nevry (2012), le piment est consommé quotidiennement à $87 \%$ par les hommes et 97,16 \% par les femmes. Il est aussi utilisé en médecine traditionnelle (Koffi-Nevry et al., 2012). Dans l'industrie pharmaceutique, il est exploité pour ses propriétés anti-oxydantes et antibiotiques (Koffi et al., 2014 ; Kouassi et al., 2016).

Par ailleurs, le piment est l'une des cultures de diversification des exportations agricoles en Côte d'Ivoire, du fait de son prix qui est de plus en plus attrayant.

Toutefois, la production du piment est limitée par de nombreux facteurs, notamment les attaques des ravageurs (Akesse et al., 2015), les maladies (Konaté \& Traoré, 1999 ; Fondio et al., 2016), la sécheresse et la réduction de la fertilité des sols agricoles. Pour faire face à cette situation, plusieurs variétés et lignées dites sélectionnées sont introduites en Côte d'Ivoire. La recherche locale, à son tour, a sélectionné parmi ces variétés et lignées dites sélectionnées, celles les mieux adaptées aux conditions locales de pratiques agricoles et, par conséquent, qualifiées de variétés et lignées ivoiriennes. Ainsi, certaines de ces variétés et lignées ivoiriennes évoluent selon des zones culturales spécifiques (soit forêt, soit savane) (CNRA, 2019). Cela découle de la diversité agro-morphologique considérable potentiellement exploitable en sélection (Sangare et al., 2009).

Par ailleurs, en termes de piment, Kouassi et Koffi-Nevry (2012) ont identifié sur les marchés d'Abidjan six variétés alors que le Centre National de Recherche Agronomique (CNRA) a publié six lignées et un cultivar local (CNRA, 2019). Il en découle ainsi que, en Côte d'Ivoire, il existerait encore des variétés de piment qui ne sont pas connues. Ce qui sous-entend que les stratégies ou schémas de sélection vis-à-vis du piment sont moins efficaces. Ainsi, l'objectif de la présente étude était de déterminer la diversité phénologique et morphologique de neuf variétés de piment dans la région du Haut-Sassandra. 


\section{Matériel et méthodes}

\subsection{Matériel végétal}

Le matériel végétal étudié était constitué de 405 plantes de neuf variétés de piment cultivées dans la région du Haut-Sassandra, à raison de 45 plantes par variété. Ces variétés sont Bec d'oiseau, Gros-Gros, Fros-Gros Ferké, Inconnu, Jaune-Jaune, Petit de Gros-Gros, Piment baoulé, Sen bon et Sen bon africain.

\subsection{Méthodes}

\subsubsection{Zone d'étude}

Les variétés de piment étudiées ont été collectées dans les quatre départements (Daloa, Issia, Vavoua et Zoukougbeu) de la région du Haut Sassandra au Centre - Ouest de la Côte d'Ivoire (Figure 1). Cette région est située entre $6^{\circ}$ et $7^{\circ}$ de latitude Nord et $7^{\circ}$ et $8^{\circ}$ de longitude Ouest.

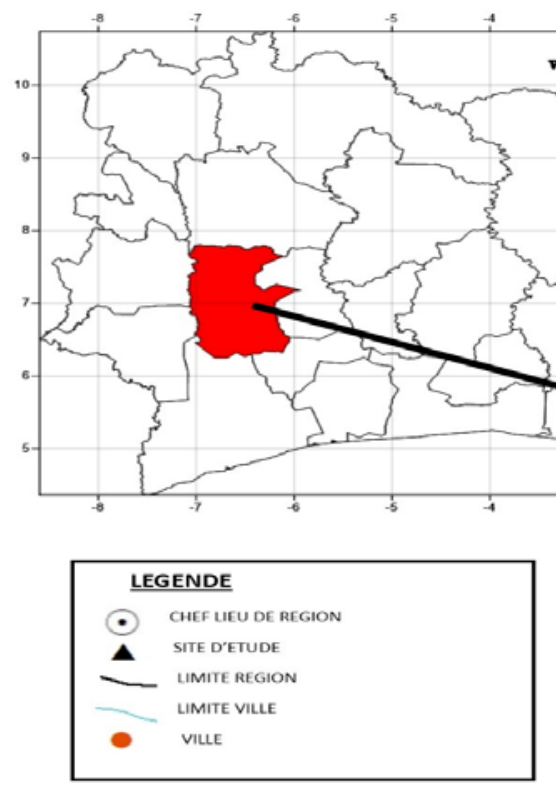

Figure 1. Carte de la Côte d’Ivoire avec indication des départements de variétés de piment étudiées

collecte des

Le climat est de type tropical humide avec deux saisons pluvieuses et deux saisons sèches (N'Guessan et al., 2014). La pluviométrie annuelle oscille entre 1200 et $1600 \mathrm{~mm}$. Les précipitations sont réparties sur toute l'année avec un maximum en juin et juillet et un minimum entre décembre et mars. La température moyenne annuelle varie de 24,65 à $27,75^{\circ} \mathrm{C}$ (Ayolié et al., 2016). Les sols sont majoritairement ferralitiques typiques, allant de moyennement à 
faiblement dénaturés. Ils sont très profonds $(20 \mathrm{~m})$ et riches en matière organique (Soro et al., 2015). La région est majoritairement couverte de forêt semi-décidue, et de savanes herbeuses et arbustives.

\subsubsection{Collecte des différentes variétés de piment}

Des fruits mûrs et frais ont été collectés lors d'un seul passage, en septembre 2019, sur les marchés où le piment est rencontré notoirement avec une diversité morphologique potentielle. Ils ont été conservés dans des seaux en plastique pendant 10 jours dans une petite chambre. Ce processus avait pour objectif de parachever la maturation physiologique des graines favorisant ainsi leur germination et la vigueur ultérieure des plantules (Segnou et al., 2012a). Les graines, issues d'égrainage des fruits, ont été séchées au soleil pendant une journée et conservées dans un endroit frais.

\subsubsection{Caractéristiques climatiques du site expérimental}

L'essai expérimental a été installé sur le site expérimental de l'Université Jean Lorougnon Guédé à Daloa. En cette année 2019, la pluviométrie a été de 246,63 mm, la température de $26,32^{\circ} \mathrm{C}$ et l'humidité atmosphérique moyennes a atteint 82,75 .

\subsubsection{Mise en place de l'essai Pépinière}

La pépinière a été réalisée, d’octobre à décembre 2019. Les graines de chaque variété, après avoir été sélectionnées par suite de test de flottaison, ont été semées à raison de 5 par trou, sous une ombrière de $80 \mathrm{~cm}$ de haut, construite à l'aide de palmes. Un arrosage quotidien à l'eau ordinaire a été assuré. Le démariage à deux plantules a été effectué 14 jours après le semis. L'ombrière a été enlevée progressivement à partir du $15^{\mathrm{e}}$ jour après semis jusqu'à ce qu'elle ait été totalement éliminée sept jours avant le repiquage.

\section{Préparation de la parcelle expérimentale}

Pour la mise en place de l'expérimentation, 27 billons de 7,5 $\mathrm{m}$ de long, 0,30 $\mathrm{m}$ de large et $0,30 \mathrm{~m}$ de haut ont été réalisés sur une parcelle défrichée. L'écartement entre ces billons a été de 0,8 $\mathrm{m}$. Une bordure de $1 \mathrm{~m}$ de large a été établie. Ainsi, la parcelle expérimentale est de $8,4 \mathrm{~m}$ de large et $24,5 \mathrm{~m}$ de long, soit une superficie de 205,8 $\mathrm{m}^{2}$.

\section{Repiquage des plantules issues de la pépinière}

Des plantules choisies au hasard en pépinière ont été transplantées sur les billons, 45 jours après semis, suivant un dispositif en blocs de Fisher (blocs aléatoires complets) (Dagnelie, 2000) de neuf traitements en trois répétitions. Les blocs ont été distancés de $1 \mathrm{~m}$. Chaque bloc comportait 9 billons 
représentant, au hasard, chacune des neuf variétés. Chaque billon a porté 15 plantes de la même variété à intervalle de $0,5 \mathrm{~m}$, soit 135 plantes par bloc et 405 plantes sur les trois blocs de l'essai.

\section{Entretien de la parcelle}

Un arrosage quotidien (matin et soir) à l'eau ordinaire et un désherbage manuel, une fois toutes les deux semaines, ont été assurés jusqu'à la fin de l'expérience.

\subsubsection{Collecte des données}

Les données collectées ont concerné la germination des semences et les paramètres morphologiques de croissance et de développement des plantes. Pour la germination des semences, le délai (Dger) et le taux (Tger) ont été considérés. Concernant les paramètres morphologiques, les variables observées et mesurées à la floraison sur chacune des 405 plantes ont été le diamètre au collet (DiaC), la hauteur de la plante (HauP), le nombre de ramifications de la tige principale (Nram), la longueur de la ramification la plus développée (LonRD), l'envergure de la frondaison (EnvFron), la surface foliaire (SurFo) et la densité du feuillage (DenFeu). Cette densité est notée sur une échelle allant de 1 à 3 , avec 1 = faible densité, 2 = densité moyenne et $3=$ forte densité.

\subsubsection{Analyse des données}

Les corrélations linéaires de Pearson entre les variables ont été calculées. Les données collectées ont été soumises à une analyse de variance. Le Modèle Linéaire Général (GLM) a été utilisé pour tester à $5 \%$ l'effet variété sur les huit variables. Dans le cas où l'analyse a été significative, un regroupement homogène des moyennes des valeurs obtenues par variable et par variété a été réalisé par le test HSD (Honestly Significant Difference) de Tukey au seuil de $5 \%$. Une analyse en composantes principales (ACP) normée centrée - réduite et une classification ascendante hiérarchique (CAH), utilisant la distance euclidienne et la méthode de Ward comme critères de distance et d'agrégation, ont été effectuées pour mettre en évidence la structure de la diversité des piments. Ces calculs et analyses ont été réalisés avec le logiciel Statistica version 7.1.

\section{Résultats}

\subsection{Répartition des variétés répertoriées}

Les neuf variétés de piment recensées et étudiées sont réparties selon le tableau 1. A l'exception du département de Zoukougbeu où trois variétés ont été répertoriées, chacun des trois autres départements a présenté cinq variétés. L’analyse du tableau 1 montre que chacune des variétés «Bec 
d'oiseau» et «Gros-Gros» a été rencontrée, dans chacun des quatre départements. Ce qui n’a pas été le cas des variétés «Sent bon africain», «Piment baoulé», «Jaune-Jaune» et «Inconnu» qui ont été uniquement et respectivement rencontrées à Vavoua, Zoukougbeu, Vavoua et Daloa.

Tableau 1. Variétés de piment recensées dans les différents départements du HautSassandra

\begin{tabular}{ll}
\hline \multicolumn{1}{c}{ Variétés } & \multicolumn{1}{c}{ Départements } \\
\hline Bec d'oiseau & Daloa, Issia, Vavoua, Zoukougbeu \\
Gros-Gros & Daloa, Issia, Vavoua, Zoukougbeu \\
Gros-Gros Ferké & Daloa, Issia \\
Inconnu & Daloa \\
Jaune-Jaune & Vavoua \\
Petit de Gros-Gros & Issia, Vavoua \\
Piment baoulé & Zoukougbeu \\
Sent bon & Daloa, Issia \\
Sent bon africain & Vavoua \\
\hline
\end{tabular}

\subsection{Corrélations entre les variables}

L'analyse du tableau 2 montre que le diamètre au collet (DiaC) a été positivement et fortement corrélé à la hauteur de plante (HauP), au nombre de branches de la tige (Nram) et à la densité du feuillage (DenFeu). Il en a été de même de la densité du feuillage avec la hauteur de plante et le nombre de branches.

\subsection{Comparaison des variétés}

\subsubsection{Germination des semences}

Pour chacune des neuf variétés, le taux de germination (Tger) a été 100 $\%$. Relativement au délai de germination (Dger), l'analyse de variance a montré des différences significatives $(p<0,001)$ entre les variétés (Tableau $3)$. Ainsi, le test HSD a rangé les neuf variétés en cinq groupes qui sont i) les variétés «Inconnu» et «Petit de Gros-Gros», ii) la variété «Gros-Gros Ferké», iii) les variétés «Sent bon africain», «Gros-Gros», «Jaune-Jaun» et «Sent bon», iv) la variété «Bec d’oiseau» et v) la variété «Piment baoulé».

Par ailleurs, les semences des variétés «Inconnu» et «Petit de Gros-Gros» ont germé dans un délai plus court que celui de la variété «Piment baoulé», respectivement en moyenne de 5 à 6 et de 8,58 jours après semis (JAS).

Tableau 2. Corrélations linéaires de Pearson entre les variables considérées.

\begin{tabular}{cccccccc}
\hline Variables & Dger & DiaC & HauP & Nram & LonRD & EnvFron & SurFo \\
\hline Dger & 1,000 & & & & & \\
Diac & $-0,288$ & 1,000 & & & & & \\
\hline
\end{tabular}




\begin{tabular}{cccccccc}
\hline HauP & $-0,510$ & $\mathbf{0 , 9 1 4 * * * *}$ & 1,000 & & & & \\
Nram & $-0,205$ & $\mathbf{0 , 8 9 4 * * *}$ & $\mathbf{0 , 7 5 0 * *}$ & 1,000 & & & \\
LonRD & 0,382 & 0,484 & 0,186 & 0,606 & 1,000 & & \\
EnvFron & $-0,144$ & 0,611 & 0,645 & 0,536 & 0,254 & 1,000 & \\
SurFo & 0,248 & $-0,203$ & $-0,221$ & $-0,365$ & $-0,052$ & 0,459 & 1,000 \\
DenFeu & $-0,363$ & $\mathbf{0 , 8 7 5 * * *}$ & $\mathbf{0 , 8 2 2} * * *$ & $\mathbf{0 , 9 0 4 * * * *}$ & 0,521 & $\mathbf{0 , 7 7 5 * *}$ & 0,072 \\
\hline
\end{tabular}

Dger : délai de germination, Diac : Diamètre au collet, HauP : hauteur de la plante, Nram : nombre de ramifications, LonRD : longueur de la ramification la plus développée, EnvFron : envergure de la frondaison, SurFo : surface foliaire, DenFeu : densité du feuillage. En gras, significatif à $5 \%$.

** : modérée, ${ }^{* * *}$ : forte (élevée), $* * * *$ : très forte (très élevée).

\subsubsection{Développement des plantes}

L'analyse de variance au seuil de $5 \%$ n'a montré aucune différence significative $(\mathrm{p}<0,05)$ entre les variétés, pour ce qui concerne le nombre de ramifications (Nram), la longueur de la ramification la plus développée (LonRD), l'envergure de la frondaison (EnvFron) et la densité du feuillage (DenFeu) (Tableau 3). Ce qui n'a pas été le cas du diamètre au collet (DiaC), de la hauteur de la plante (HauP) et de la surface foliaire (SurFo).

Concernant le diamètre au collet (Diac), les neuf variétés de piment ont été placées dans un seul groupe avec des diamètres moyens variant de 3,29 à 4,13 mm (Tableau 3). Quant à la hauteur de la plante (HauP), trois groupes de variétés se sont distingués: i) la variété «inconnu» présentant la valeur la plus élevée $(23,02 \mathrm{~cm})$; ii) la variété «Gros-Gros Ferké» affichant la valeur intermédiaire $(19,82 \mathrm{~cm})$ entre celle de «Inconnu» celles du troisième groupe composé des autres variétés dont les valeurs ont varié de 14,43 à 16,55 cm.

Six groupes de variétés ont été distingués au niveau de la surface foliaire (SurFo). Les groupes (i) et (ii) représentés par les variétés «Gros-Gros Ferké» et «Bec» d'oiseau» ont présenté les surfaces moyennes les plus élevées (20,57 
Tableau 3. Effet variété de piment sur les huit variables phénologique et morphologiques considérées

\begin{tabular}{|c|c|c|c|c|c|c|c|c|}
\hline \multirow[b]{2}{*}{ Variétés } & \multicolumn{8}{|c|}{ variables phénologique et morphologiques } \\
\hline & $\begin{array}{l}\text { Dger } \\
\text { (jours) }\end{array}$ & $\begin{array}{c}\text { DiaC } \\
(\mathrm{mm})\end{array}$ & $\begin{array}{c}\text { HauP } \\
(\mathrm{cm})\end{array}$ & Nram & $\begin{array}{c}\text { LonRD } \\
(\mathrm{cm})\end{array}$ & $\begin{array}{c}\text { EnvFron } \\
(\mathrm{cm})\end{array}$ & $\begin{array}{c}\text { SurFo } \\
(\mathrm{cm})\end{array}$ & DenFeu \\
\hline Bec d'oiseau & $7,46 \pm 2,32^{\mathrm{ab}}$ & $3,613 \pm 1,110^{\mathrm{a}}$ & $15,31 \pm 5,82^{b}$ & $3,13 \pm 1,93$ a & $5,11 \pm 3,80^{a}$ & $13,02 \pm 4,97^{\mathrm{a}}$ & $19,36 \pm 11,01^{\text {ac }}$ & $2,14 \pm 0,81^{\mathrm{a}}$ \\
\hline Gros-Gros & $6,37 \pm 1,56^{\mathrm{abc}}$ & $3,475 \pm 1,037^{\mathrm{a}}$ & $16,55 \pm 6,25^{b}$ & $3,35 \pm 1,79^{\mathrm{a}}$ & $4,45 \pm 3,87^{\mathrm{a}}$ & $11,69 \pm 4,25^{\mathrm{a}}$ & $13,515 \pm 8,42^{\text {bd }}$ & $2,00 \pm 0,80^{\mathrm{a}}$ \\
\hline Gros-Gros Ferké & $6,00 \pm 0,62^{\mathrm{bc}}$ & $4,028 \pm 0,916^{a}$ & $19,82 \pm 7,58^{\mathrm{ab}}$ & $3,86 \pm 2,21^{\mathrm{a}}$ & $5,52 \pm 4,14^{\mathrm{a}}$ & $14,05 \pm 3,19^{a}$ & $20,57 \pm 14,56^{\text {ab }}$ & $2,54 \pm 0,74^{\mathrm{a}}$ \\
\hline Inconnu & $5,04 \pm 1,51^{\mathrm{c}}$ & $4,137 \pm 0,659^{a}$ & $23,02 \pm 4,41^{\mathrm{a}}$ & $3,54 \pm 1,53^{\mathrm{a}}$ & $4,25 \pm 2,29^{a}$ & $11,81 \pm 2,53^{\mathrm{a}}$ & $11,62 \pm 4,39^{\mathrm{cd}}$ & $2,27 \pm 0,66^{\mathrm{a}}$ \\
\hline Jaune-Jaune & $6,43 \pm 2,34^{\mathrm{abc}}$ & $3,679 \pm 1,004^{\mathrm{a}}$ & $15,24 \pm 4,43^{b}$ & $3,50 \pm 1,87^{a}$ & $5,04 \pm 3,51^{\mathrm{a}}$ & $10,57 \pm 4,16^{\mathrm{a}}$ & $12,11 \pm 7,15^{\mathrm{bcd}}$ & $2,00 \pm 0,88^{a}$ \\
\hline Petit de Gros-Gros & $5,54 \pm 1,14^{\mathrm{c}}$ & $3,291 \pm 0,912^{\mathrm{a}}$ & $15,80 \pm 5,41^{\mathrm{b}}$ & $3,14 \pm 1,56^{\mathrm{a}}$ & $4,16 \pm 3,50^{\mathrm{a}}$ & $11,75 \pm 4,78^{\mathrm{a}}$ & $12,08 \pm 6,60^{\text {bcd }}$ & $2,04 \pm 0,84^{\mathrm{a}}$ \\
\hline Piment baoulé & $8,58 \pm 1,88^{a}$ & $3,800 \pm 1,273^{a}$ & $15,83 \pm 6,04^{\mathrm{b}}$ & $3,83 \pm 1,99^{a}$ & $6,71 \pm 4,76^{\mathrm{a}}$ & $10,42 \pm 4,27^{a}$ & $12,16 \pm 9,23^{\mathrm{bcd}}$ & $2,00 \pm 0,95^{\mathrm{a}}$ \\
\hline Sent bon & $6,86 \pm 0,38^{\mathrm{abc}}$ & $3,367 \pm 0,537^{a}$ & $14,43 \pm 3,60^{\mathrm{b}}$ & $2,00 \pm 1,92^{\mathrm{a}}$ & $2,69 \pm 2,06^{\mathrm{a}}$ & $11,43 \pm 2,51^{\mathrm{a}}$ & $17,25 \pm 6,96^{\text {abcd }}$ & $1,7 \pm 0,95^{\mathrm{a}}$ \\
\hline Sent bon africain & $6,33 \pm 1,92^{\mathrm{abc}}$ & $3,370 \pm 0,910^{\mathrm{a}}$ & $15,41 \pm 4,45^{\mathrm{b}}$ & $2,67 \pm 1,83^{\mathrm{a}}$ & $2,83 \pm 1,03^{\mathrm{a}}$ & $11,42 \pm 3,42^{\mathrm{a}}$ & $15,02 \pm 9,39^{\text {abcd }}$ & $1,75 \pm 0,74^{\mathrm{a}}$ \\
\hline $\mathrm{F}$ & 8,076 & 2,047 & 5,670 & 1,1797 & 1,5744 & 1,612 & 3,9836 & 1,6616 \\
\hline $\mathrm{P}$ & $<0,001$ & 0,041938 & $<0,001$ & 0,312074 & 0,133264 & 0,122150 & $<0,001$ & 0,108671 \\
\hline
\end{tabular}

Dger : délai de germination, Diac : Diamètre au collet, HauP : hauteur de la plante, Nram : nombre de ramifications, LonRD : longueur de la ramification la plus développée, EnFron : envergure de la frondaison, SurFo : surface foliaire, DenFeu : densité du feuillage, mm : millimètre, cm : centimètre, $\mathrm{cm}^{2}$ : centimètre carré, $\mathrm{F}$ : valeur du test de Fischer, $\mathrm{p}$ : probabilité associée au test de Fischer.

Les valeurs moyennes dans une colonne suivies de la même lettre ou des mêmes lettres ne diffèrent pas significativement à 5 \% selon le test de Fisher. 


\section{ESJ Natural/Life/Medical Sciences}

et 19,36 $\mathrm{cm}^{2}$ ). Les variétés «Sent bon» et «Sent bon africain» formant le groupe (iii) et «Gros-Gros» représentant le groupe (iv) ont eu les valeurs respectives $17,25,15,02$ et $13,515 \mathrm{~cm}^{2}$. Le groupe (v) est formé par les variétés «Piment baoulé», «Jaune-Jaune» et «Petit de Gros-Gros» ont présenté des valeurs ayant varié de 2,08 à 12,16 $\mathrm{cm}^{2}$. La variété «Inconnu» constituant le groupe (vi) a eu la plus faible valeur moyenne $\left(11,62 \mathrm{~cm}^{2}\right)$ (Tableau 3).

\subsection{Diversité des variétés par l'analyse en composantes principales}

Les plans $(1,2)$ et $(1,3)$ de l'analyse en composantes principales (ACP) ont expliqué, respectivement, 75,79 et 73,07 \% de l'inertie totale (Figure 2). La diversité des neuf variétés de piment est analysée sur ces deux plans.
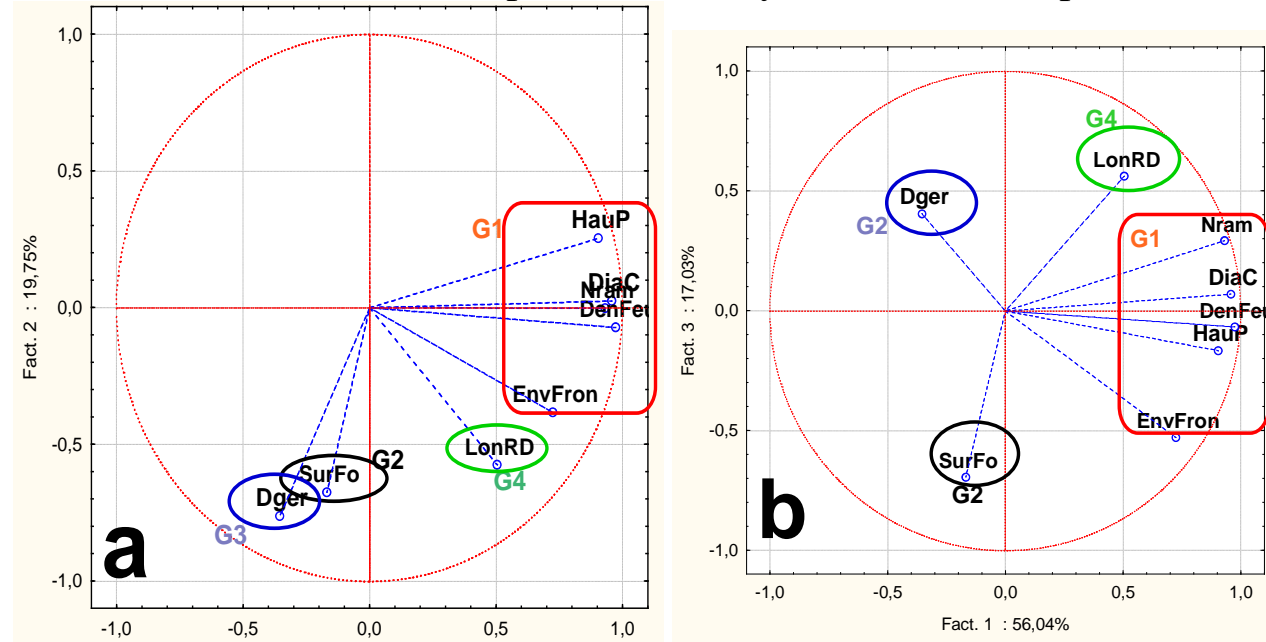

Figure 2. Cercles des corrélations des variables dans les plans 1,2 (a) et 1,3

(b) de l'analyse en composantes principales et les différents Groupes distingués.

Dger : délai de germination, Diac : Diamètre au collet, HauP : hauteur de la plante, Nram : nombre de ramifications, LonRD : longueur de la ramification la plus développée, EnFron : envergure de la frondaison, SurFo : surface foliaire, DenFeu : densité du feuillage,

Les corrélations entre les variables étudiées et les axes factoriels, d'une part, et les contributions de ces variables aux axes, d'autre part, sont présentées dans le tableau 4. Il ressort de ce tableau que les variables DiaC, HauP, Nram et DenFeu ont été très fortement et positivement corrélés $(0,905 \leq \mathrm{r} \leq 0,973)$ à l'axe 1. Ces variables ont contribué à la formation de la partie positive de l'axe 1. Aussi, le Dger et la SurFo ont été négativement, faiblement à modérément corrélés $(-0,762 \leq \mathrm{r} \leq-0,676)$ à l'axe 2 . Le Dger a contribué à la formation de la partie négative de cet axe ; la SurFo y participe à un degré moindre. Cette SurFo a aussi été négativement et quasi-modérément corrélée $(r=-0,692)$ à l'axe 3, dont elle caractérise la partie négative. Conséquemment, 
quatre groupe (G1, G2, G3, G4) de variables ont été distingués (Figures 2a et 2b). Le groupe 1 (G1) a été composé de DiaC, HauP, Nram, EnvFron, DenFeu. Le groupe 2 (G2) a été représenté par SurFo, le groupe 3 (G3) par Dger et le groupe 4 (G 4) par LonRD.

Tableau 4. Correlations entre variables et axes et contribution des variables aux axes

\begin{tabular}{|c|c|c|c|c|c|c|}
\hline \multirow[t]{2}{*}{ Variables } & \multicolumn{3}{|c|}{ Corrélations entre variables et axes } & \multicolumn{3}{|c|}{$\begin{array}{c}\begin{array}{c}\text { Contributions des variables à la formation des } \\
\text { axes }\end{array} \\
\end{array}$} \\
\hline & axe 1 & axe 2 & axe 3 & axe 1 & axe 2 & axe 3 \\
\hline Dger & $-0,545^{*}$ & $-0,762 * *$ & 0,407 & 0,028027 & 0,367663 & 0,121337 \\
\hline Diac & $0,957 * * * *$ & 0,025 & 0,071 & 0,204414 & 0,000382 & 0,003659 \\
\hline HauP & $0,905^{* * * *}$ & 0,254 & $-0,166$ & 0,182645 & 0,040965 & 0,020256 \\
\hline Nram & $0,931 * * * *$ & $-0,002$ & 0,293 & 0,193284 & 0,000002 & 0,063119 \\
\hline LonRD & $0,505^{*}$ & $-0,573^{*}$ & $0,562 *$ & 0,056944 & 0,207747 & 0,232132 \\
\hline EnvFron & $0,729 * *$ & $-0,383$ & $-0,528 *$ & 0,117109 & 0,092827 & 0,204625 \\
\hline SurFo & $-0,168$ & $-0,676^{*}$ & $-0,692 *$ & 0,006307 & 0,287272 & 0,351638 \\
\hline DenFeu & $0,973 * * * *$ & $-0,070$ & $-0,066$ & 0,211270 & 0,003143 & 0,003184 \\
\hline
\end{tabular}

Dger : délai de germination, Diac : Diamètre au collet, HauP : hauteur de la plante, Nram : nombre de ramifications, LonRD : longueur de la ramification la plus développée, EnFron : envergure de la frondaison, SurFo : surface foliaire, DenFeu : densité du feuillage, *: faible, ${ }^{* *}$ :modérée, ${ }^{* * * *}$ : très forte (très élevée). En gras, les valeurs des paramètres ayant contribué aux axes.

Les variétés «Inconnu» et «Sent bon» ont été très bien représentées sur l’axe 1 , avec respectivement de fortes contributions de 46,991 et de 38,570\% (Tableau 5). «Bec d'oiseau» a contribué à l'axe 2 pour 48,131 \%. Sur l’axe 3, «Piment baoulé» a été assez bien représentée pour une contribution de 55,810 $\%$.

Tableau 5. Contributions des variétés aux axes factoriels

\begin{tabular}{lccc}
\hline Variétés & axe 1 & axe 2 & axe 3 \\
\hline Bec d'oiseau & 0,40401 & 48,13084 & 9,96852 \\
Gros-Gros & 0,07523 & 0,02554 & 0,04686 \\
Gros-Gros Ferké & 2,87133 & 3,67286 & 8,27856 \\
Inconnu & 46,99119 & 12,10337 & 4,97513 \\
Jaune-Jaune & 0,01431 & 7,94429 & 13,00707 \\
Petit de Gros-Gros & 0,00886 & 6,46849 & 0,71431 \\
Piment baoulé & 0,15161 & 12,98174 & 55,81037 \\
Sent bon & 38,57042 & 1,01913 & 6,35175 \\
Sent bon africain & 10,91303 & 7,65374 & 0,84741 \\
\hline
\end{tabular}

Cinq groupes (GA, GB, GC, GD, GE) de variétés de piment ont été distingués (Figures 3). Le groupe A (GA) est représenté par «Inconnu». Le groupe B (GB) est composé de «Sent bon» et «Sent bon africain», le groupe C (GC) de «Bec d'oiseau » et «Gros-Gros Ferké». Le groupe D (GD) s'est identifié à «Piment baoulé», tandis que le groupe E (GE) est composé de «Gros-Gros », «Petit de Gros-Gros» et de « Jaune jaune ». 

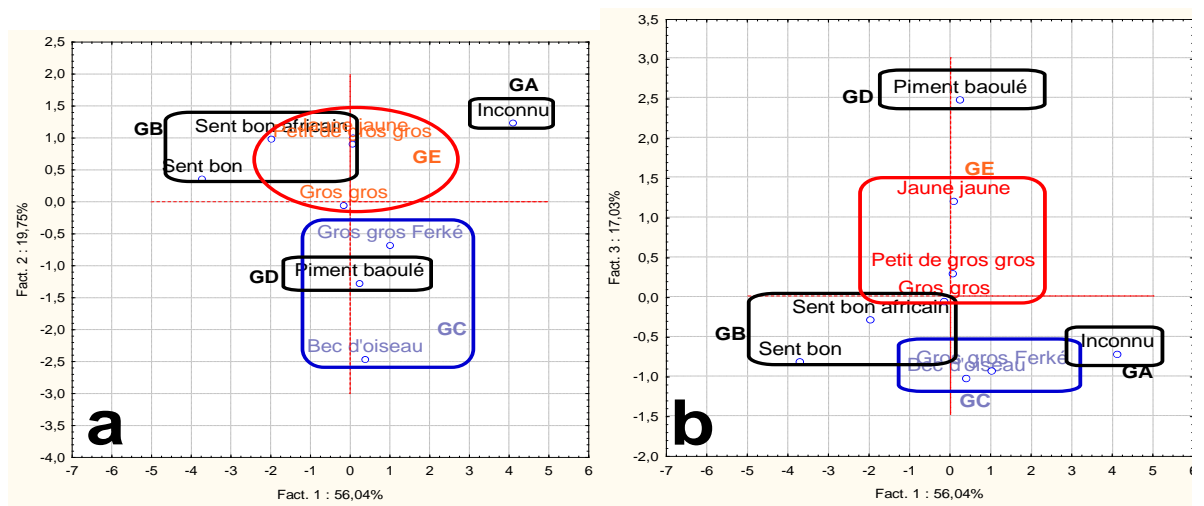

Figure 3. Groupes des neuf variétés de piment dans deux plans d'analyse en composantes principales

a : plan $(1,2)$; b : plan $(1,3)$

\subsection{Diversité des variétés par la classification ascendante hiérarchique}

La diversité par la classification ascendante hiérarchique (CAH) des neuf variétés de piment étudiées est présentée à la figure 4 . La troncature effectuée à la distance 5 a dégagé quatre principales classes $(\mathrm{C} 1, \mathrm{C} 2, \mathrm{C} 3, \mathrm{C} 4)$ de variétés. La classe 1 s'est identifiée à « Inconnu ». La classe 2 est composée de « Sent bon » et «Sent bon africain », tandis que la classe 3 s'est identifiée à « Bec d'oiseau » et à «Gros gros Ferké». La classe 4, quant à elle, est composée de «Petit de gros-Gros», «Jaune-Jaune», «Gros-Gros» et de «Piment baoulé».

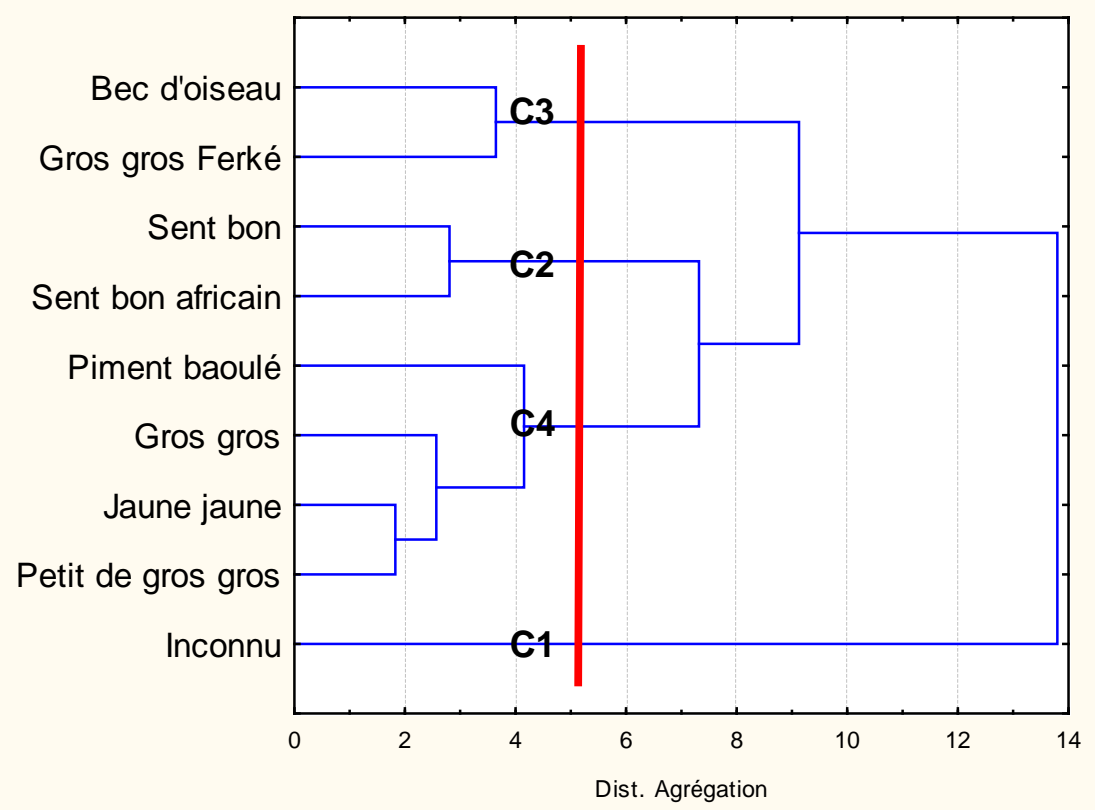

Figue 4. Classes de variétés de piment distinguées sur le dendrogramme obtenu par application de la méthode de Ward et de la distance

Euclidienne 


\section{Discussion}

La connaissance de la diversité génétique est essentielle pour l'utilisation efficiente de celle-ci en amélioration variétale. Cette connaissance passe par la description des ressources génétiques. Les caractéristiques phénologiques et morphologiques sont les premiers candidats à cette description. Une évaluation a, alors, été conduite pour déterminer la diversité phénologique et morphologique de neuf variétés de piment répertoriées à partir d'accessions collectées dans les quatre départements de la région du Haut-Sassandra. Ces neuf variétés n’ont pas été rencontrées, toutes, dans chaque département. Une pareille disparité présentielle de variétés est constatée chez plusieurs espèces cultivées (Look et al., 2013; Touré et al., 2013; Gbaguidi et al., 2015). L’absence d'une variété donnée dans un département serait due soit à sa non culture, soit au fait que ses fruits n'étaient pas disponibles pendant la collecte, soit encore au fait qu'elle est connue sous différents noms d'un site à un autre. Dans ce dernier cas, la connaissance de tous les noms s'avère indispensable. La pluralité de noms de variétés a déjà été rapportée. Cela est le cas chez le voandzou dont les noms locaux attribués aux morphotypes varient en fonction du groupe et du sous groupe ethnique (Touré et al., 2013) et le niébé (Gbaguidi et al., 2015). Par ailleurs, une accession de Daloa, sans nom de variété, a été appelée «Inconnu» dans cette étude. Ce cas confirme le fait qu'il y a des variétés de piment qui demeurent inconnues des utilisateurs.

L'analyse de variance a ressorti trois à six groupes homogènes de variétés pour le délai de germination, la hauteur de la plante et la surface foliaire. Ces résultats témoignent d'une forte variabilité phénologique et morphologique entre ces neuf variétés. Le délai de germination, la hauteur de la plante et la surface foliaire ont été les caractères les plus discriminants. Des résultats semblables ont été obtenus par de nombreux auteurs chez le piment (Segnou et al., 2012a, 2012b ; Zhang Xiao-min et al., 2016; Andrade et al., 2020; Waongo et al., 2021).

L'importante variabilité observée dans cette étude contraste avec le principe que la variabilité génétique est faible au sein d'une zone géographique réduite. (Ravindran et al., 2005). En effet, les variétés étudiées ont été acquises à l'issue d'une collecte sur les marchés de quatre villes d'une même région de superficie relativement réduite. Ainsi donc, cette variabilité serait due à plusieurs faits. Le piment est l'une des cultures à variabilité potentielle élevée (Djidji, 1995). Cette variabilité est renforcée par les introductions de variétés diversifiées à partir d'autres pays et par les créations variétales pour répondre aux exigences multiples des utilisateurs (Fondio et al., 2015; CNRA, 2019). Aussi, elle est générée par les pratiques de production, notamment les échanges de semences entre agriculteurs (Delaunay et al., 2008; Missihoun et al., 2012) et la culture polyvariétale qui consiste à cultiver plusieurs variétés 
dans un même champ. Cette dernière pratique engendre d'autres génotypes, étant donné que le piment se multiplie essentiellement de façon générative et que, par ailleurs, l'autofécondation et la fécondation croisée existent naturellement chez le genre Capsicum (Waongo et al., 2021).

L'analyse en composantes principales (ACP) sur les trois premiers axes représentant 92,82 \% de la variabilité totale a permis d'identifier cinq groupes. Pla (1986) a suggéré au moins 80 \% de la variabilité totale. La classification ascendante hiérarchique (CAH) a rangé les variétés «Piment baoulé», «GrosGros», «Petit de Gros-Gros» et «Jaune-Jaune» dans un seul et même groupe et établi quatre groupes dont trois sont identiques aux groupes formés par ACP. Ces groupes de variétés se distinguant par le délai de germination, la hauteur de la plante, la surface foliaire. Les nombres de groupes de génotypes ou d'accessions obtenus par les méthodes multivariées reflètent les niveaux de variabilité des germoplasmes. Ils peuvent dépendre des outils et des effectifs. Ainsi, sur la base de paramètres de croissance végétative tels que ceux utilisés dans la présente étude à savoir le délai de germination, le diametre de la tige, la hauteur de la plante, les dimensions de la feuille, la largeur de la canopée, trois à neuf groupes ont été obtenus avec 18 à 192 génotypes ou accessions (Segnou et al., 2012b; Ballina-Gómez et al., 2013; Nsabiyera et al., 2013; Andrade et al., 2020 ; Bianchi et al., 2020: Waongo et al., 2021). Comparativement à ces travaux, les neuf variétés de cette étude, réparties en quatre groupes, présentent un niveau élevé de variabilité.

Par ailleurs, les analyses ont mis en évidence trois tendances de variétés aux caractéristiques opposées. Une tendance englobe les semences ayant germé dans un court délai et engendré des plantes de plus grands diamètres et hauteurs. C’est le cas de la variété «Inconnu» dont les caractéristiques sont les plus différentes de celles des autres variétés. Une autre concerne les semences ayant germé dans un long délai et engendré des plantes de plus faibles diamètres et hauteurs, avec des surfaces foliaires, nombres de ramifications, longueurs de la ramification la plus développée, envergures de frondaison densités de feuillage moyens. Les variétés «Piment baoulé», «Gros-Gros», «Jaune-Jaune» et «Petit de Gros-Gros» se retrouvent dans cette tendance. Entre ces deux tendances se retrouvent les combinaisons intermédiaires de caractéristiques. Ces résultats sont semblables à ceux de Nsabiyera et al. (2013) sur 37 génotypes de Capsicum annum d'Ouganda et de Bianchi et al. (2020) sur 56 accessions de Capsicum chinense du Brésil et du Pérou. De plus, Nsabiyera et al. (2013) ont observé que les variétés locales sont de grandes tailles, de grands diamètres de tige et de larges canopées et le contraire chez les variétés exotiques améliorées et introduites. Selon ces observations, la variété «Inconnu» pourrait être une variété locale et les variétés «Gros-Gros», «Jaune-Jaune» et «Petit de Gros-Gros» des variétés améliorées. 
Les caractères phéno-morphologiques ont permis de discriminer les variétés étudiées. En dépit des influences environnementales, la diversité observée pourrait résulter d'une variation dans la constitution génétique des variétés (N`dri et al., 2011). Ces caractères sont donc utiles en caractérisation. En effet, il existe une relation directe entre la croissance végétale et le développement, la surface foliaire, la photosynthèse, la floraison et la fructification (Silva et Silva, 2021 ; Waongo et al., 2021)

\section{Conclusion}

Neuf variétés de piment cultivées dans la région du Haut-Sassandra ont été évaluées pour leur diversité phénologique et morphologique. Elles ont présenté une importante diversité, notamment au niveau du délai de germination, de la hauteur de la plante et de la surface foliaire. Par ailleurs, la structuration de cette diversité a opposé ces variétés. Ainsi, la variété «Inconnu», avec le délai de germination le plus court, la hauteur de plante la plus élevée, la meilleure croissance latérale et la densité du feuillage la plus grande, a été la plus différèrent des autres. Aussi, les variétés «Piment baoulé», «Gros-Gros», «Jaune-Jaune» et «Petit de Gros-Gros» ont présenté un long délai de germination, des plantes de plus faibles diamètres et hauteurs, des surfaces foliaires, des nombres de ramifications, des longueurs de la ramification la plus développée, des envergures de frondaison, et des densités de feuillage moyens. Les variétés «Bec d'oiseau», «Gros-Gros Ferké», «Sent bon» et «Sent bon africain» ont présenté des combinaisons intermédiaires de valeurs des paramètres. Il découle de ces résultats que les paramètres phénologiques et morphologiques sont utiles pour la caractérisation des germoplasmes.

\section{References:}

1. Akesse, E. N., Ouali-N'goran, S-W. M., \& Tano, Y. (2015). Insectes ravageurs du piment Capsicum chinense Jacq. (Solanaceae) à Port-Bouët (Abidjan-Côte d'Ivoire) : Pratiques de lutte par les pesticides chimiques. Journal of Applied Biosciences, 93, 8667-8674.

2. Andrade, P. J. N., Monteros-Altamirano, A., Bastidas, C. G. T., \& Sørensen, M. (2020). Morphological, Sensorial and Chemical Characterization of Chilli Peppers (Capsicum spp.) from the CATIE Genebank. Agronomy, 10(1732), 1-18.

3. Ayolié, K., Kouassi, N. J., Angaman, D.M., Kouadio, A. B., Soro, D., Kouakou, T. H. \& Kouadio, Y. J. (2016). Effect of water stress on morphological, physiological and biochemical parameters of three varieties of tomato (Lycopersicon esculentum Mill.). cultivated in Côte d'Ivoire, International Journal of Scientific Engineering and Applied Science, 2(11), 148-161. 
4. Ballina-Gómez, H., Latournerie-Moreno, L., Ruiz-Sánchez, E., PérezGutiérrez, A., \& Rosado-Lugo, G. 2013. Morphological characterization of Capsicum annuum L. accessions from southern Mexico and their response to the Bemisia tabaci-Begomovirus complex. Chilean Journal Of Agricultural Research, 73(4), 329-338.

5. Bianchi, P. A., Almeida da Silva, L. R., Alencar André da Silva, A., Araújo, S. P., Diniz, E., Pimenta, S., Sudré, C. P., Erpen-Dalla, C., Azeredo, G. L. S., \& Rosana, R. (2020). Biomorphological Characterization of Brazilian Capsicum Chinense Jacq. Germplasm. Agronomy, 10(447), 1-17.

6. CNRA, (2019). Répertoire des variétés améliorées de cultures vivrières. Edition 2019. Direction de la Recherche scientifique et de l'appui au développement et Direction des Innovations et des systèmes d'information, Centre National de Recherche Agronomique (CNRA), Abidjan, Côte d'Ivoire, 80 p.

7. Dagnelie, P. (2000). La planification des expériences: choix des traitements et dispositifexpérimental. Journal se la Société française de Statistique, 141(1 \& 2), 5-29.

8. Delaunay, S., Tescar R-P., Oualbego A., vom Brocke K., \& Lançon J. (2008). La culture du coton ne bouleverse pas les échanges traditionnels de semences de sorgho. Cahiers Agricultures, 17, 189- 194.

9. Djidji, A. H. (1995). La collection de germpolasme des plantes légumieres en Cote d'Ivoire. Rapport d'étude. RADHORT Documents. RADHORT, 6 p.

10. Eching El-Ghoraba, A. H., Javedb, Q,, Anjumb, F. M., Hamedc, S. F., \& Shaabana, H. A. (2013). Pakistani Bell Pepper (Capsicum annum L.): Chemical Compositions and its Antioxidant Activity. International Journal Food Properties, 16(1), 18-32.

11. Fondio. L., N'zi, J.-C., \& Kobenan, K. (2015). Comportement agronomique et sanitaire de nouvelles lignées de piment (Capsicum sp) dans le Sud de la Côte d'Ivoire. Journal of Applied Biosciences, 92, 85948609.

12. Fondio, L., Kouamé, C., Djidji, H. A., \& Aïdara, S. (2016). Bien cultiver le piment en Côte d'Ivoire. Fiche Technique. Direction des Programmes de Recherche et de l'Appui au Développement -Direction des innovations et des systèmes d'information. CNRA, Abidjan, Côte d'Ivoire, 4 p.

13. Gbaguidi, A. A., Assogba, P., Dansi, M., Yedomonhan, H., \& Dansi, A. A. (2015). Caractérisation agromorphologique des variétés de niébé cultivées au Bénin. International Journal of Biological and Chemical Sciences, 9(2), 1050-1066. 
14. Koffi, A. C., Koffi-Nevry, R., Kouassi, K. C., \& Loukou, Y. G. (2014). Activité des extraits de six variétés de piment (Capsicum) utilisés en Côte d'Ivoire. Journal of Applied Biosciences, 82, 7379 -7388.

15. Koffi-Nevry, R., Kouassi, C., Nanga, Z., Koussémon, M., \& Loukou, G, (2012). Antibacterial activity of two bell pepper extracts: Capsicum annum L. and Capsicum frutenscens. International Journal of Food Properties, 15, 961-971.

16. Konaté, G. \& Traoré, O. (1999). Caractérisation et distribution du virus de la panachure du poivron en Afrique de l'Ouest. Cahiers Agricultures, 8, 132-134.

17. Kouassi, K. C., \& Koffi-Nevry, R. (2012). Evaluation de la connaissance et utilisation des variétés de piment (Capsicum) cultivées en Côte d'Ivoire. International Journal of Biological and Chemical Sciences, 6(1), 175-185.

18. Kouassi, K. C., Coulibaly, B., Coulibaly, I., Koffi A. C., \& Koffi-Nevry, R. (2016). Antimicrobial Activity of the Varieties of Peppers (Capsicum) of Côte d'Ivoire on Multiresistant Strains. International Journal of Current Microbiology and Applied Sciences, 5(10), 875-890.

19. Loko, Y. L., Dansi, A., Linsoussi, C., Tamo, M., Vodouhè, R., Akoegninou, A., \& Sanni, A. (2013). Current status and spatial analysis of Guinea yam (Dioscorea cayenensis Lam. -D. rotundata Poir. complex) diversity in Benin. International Research Journal of Agricultural Science and Soil Science, 3(7), 219-238.

20. Missihoun, A.A., Agbangla, C., Adoukonou-Sagbadja, H., Ahanhanzo, C. \& Vodouhè, R. (2012). Gestion traditionnelle et statut des ressources génétiques du sorgho (Sorghum bicolor L. Moench) au Nord-Ouest du Bénin. International Journal of Biological Chemical Sciences, 6, 10031018.

21. N’Dri, A. A. N.., Vroh-Bi, I., Kouamé, P. L., \& .Zoro, Bi I. A. (2011). Bases génétiques et biochimiques de la capacité germinative des graines: implications pour les systèmes semenciers et la production alimentaire. Sciences \& Nature. 8(1), 119-137.

22. N’Guessan, A. H., N’Guessan, K. F., Kouassi, K. P., Kouamé, N. N., \& N'Guessan, P. W. (2014). Dynamique des populations du foreur des tiges du cacaoyer, Eulophonotus myrmeleon Felder (Lépidoptère : Cossidae) dans la région du Haut-Sassandra en Côte d'Ivoire. Journal of Applied Biosciences, 83, 7606-7614.

23. Nsabiyera, V., Logose, M., Ochwo-Ssemakula, M., Sseruwagi, P., Gibson, P., \& Ojiewo, C. (2013). Morphological Characterization of Local and Exotic Hot Pepper (Capsicum annuum L.) Collections in Uganda. Bioremediation, Biodiversity and Bioavailability, 7(1), 22-32. 
24. Ravindran, P. N., Babu, K. N., \& Shiva, K. N. (2005). Botany and Crop Improvement of Ginger. In P.N. Ravindran \& K.N. Babu. (eds), Medicinal and aromatic plantsIndustrial profiles: Ginger, the genus Zingiber (pp.15-85). CRC Press. Washington DC.

25. Pla, E. (1986). Análisis multivariado: Método de componentes principales. 94 p. Secretaría General de la Organización de los Estados Americanos, Washington, DC, USA.

26. Sangare, A., Koffi E., Akamou, F., \& Fall, C. A. (2009). Etat des ressources phytogénétiques pour l'alimentation et l'agriculture; second rapport national. Ministère de l'Agriculture, République de Côte d'Ivoire, $64 \mathrm{p}$.

27. Segnou, J., Amougou, A., \& Youmbi, E. (2012a). Viabilité et développement végétatif des plantules de piment (Capsicum annuum L.) suivant différents matériels de conditionnement des semences. Tropicultura, 30(1), 15-23.

28. Segnou, J., Akoa A., Youmbi, E., \& Njoya, J. (2012b). Variabilité des caractères morphologiques et analyse des composantes du rendement chez le piment (Capsicum annuum 1.). Agronomie Africaine, 24(3), 183195.

29. Silva, M. R. E., \& Silva, R. N. O. (2021). Morpho-agronomic haracterization and genetic diversity in peppers (Capsicum spp.). Revista Caatinga, Mossoró, 34(3), 505-513.

30. Soro, D., Ayolié, K., Gohi Bi, Z. F., Yao, Y. F., Konan-Kan, K. H., Sidiky, B., Téhua, A. P. \& Kouadio, Y. J. (2015). Impact of organic fertilization on maize (Zea mays L.) production in a ferralitic soil of centre west côte d'ivoire. Journal of Experimental Biology and Agricultural Sciences, 3(6), 556-565.

31. Touré, Y. Koné, M., Silué, S., \& Kouadio, Y. J. (2013). Prospection, collecte et caractérisation agromorphologique des morphotypes de voandzou [Vigna subterranea (L.) VERDC. (Fabaceae)] de la zone savanicole en Côte d'Ivoire. European Scientific Journal, 9(24), 308-325.

32. Waongo, L., Kiebre, M., Sawadogo, B., Ouedraogo, M. H., Kiebre, Z., \& Bationo/Kando, P. (2021). Phenotyping of local chili pepper (Capsicum spp) accessions cultivated in Burkina Faso. International Journal of Agricultural Policy and Research, 9(1), 16-23.

33. Zhang Xiao-min, Zheng-Hai, Z., Xiao-Zhen, G., Sheng-Li, M., Xi-Xiang, L., Chadœuf, J., Palloix, A., Li-Hao, W., \& Bao-Xi, Z. (2016). Genetic diversity of pepper (Capsicum spp.) germplasm resources in China reflects selection for cultivar types and spatial distribution. Journal of Integrative Agriculture, 15(9), 1991-2001. 